\title{
THE DIFFRACTION OF ELASTIC WAVES BY SMALL CYLINDRICAL CAVITIES
}

\author{
V. T. BUCHWALD
}

(Received 24 April 1978)

\begin{abstract}
In order to use the method of asymptotic matching for low frequencies, the equations of plane elastostatics are reformulated in terms of the two scalar potentials commonly used in plane elastodynamics. It is shown that the resulting equations of plane elastostatics can be reduced to those first obtained by Muskhelishvili. The use of the formulation is illustrated by considering the case of the plane diffraction of a $\boldsymbol{P}$ wave by a circular, cylindrical cavity of small radius. The results agree with those obtained from the exact solution of the problem.
\end{abstract}

\section{Introduction}

Although the method of asymptotic matching was used many years ago, in principle, by Lord Rayleigh [4] and is now used frequently in fluid mechanics, the technique does not seem to have been generally adopted as yet to study the diffraction of elastic waves by small cylindrical obstacles. While certain specialized cases have been considered by Sabina and Willis [5] and Lutz [2], perhaps the main difficulty is that in elastodynamics we have two wave equations, and the basic equation in elastostatics is the biharmonic equation. $\uparrow$ The relationship between the two types of equation is not obvious [Sternberg, 7].

In this paper the equations of plane elastostatics are evolved in terms of the same displacement potentials that are commonly used in elastodynamics. It will be shown that the potentials satisfy generalized Cauchy-Riemann relations, leading to a complex variable formulation which is very similar to that of Muskhelishvili [3], although the latter used the Airy stress function as a starting point. The consequent theory is convenient for the asymptotic matching procedure. As an illustration, the method is used to determine the asymptotic form of the diffraction of a plane dilatational wave by a circular cylindrical cavity, the diameter of which is small compared with the length of the incident wave.

$\uparrow$ Note added in proof. The author has discovered that R. P. Kanwal (J. Math. Phys. 44 (1965), 275-283) proposed a technique similar to the one in this paper. Unfortunately, Kanwal's scheme implies that the electrostatic problem can be reduced generally to the solution of Laplace's equation, a result which is well-known to be incorrect. 


\section{Basic formulation}

Referred to plane Cartesian coordinates $\left(x^{\prime}, y^{\prime}\right)$, the linear equations of motion of an elastic solid with no body forces are

$$
\begin{gathered}
\frac{\partial}{\partial x^{\prime}} \tau_{x x}+\frac{\partial}{\partial y^{\prime}} \tau_{x y}+\rho \sigma^{2} u=0 \\
\frac{\partial}{\partial x^{\prime}} \tau_{x y}+\frac{\partial}{\partial y^{\prime}} \tau_{y y}+\rho \sigma^{2} v=0
\end{gathered}
$$

where $\tau_{x x}, \tau_{x y}, \tau_{y y}$ are the components of the stress tensor in these coordinates, $(u, v)$ is the displacement vector, $\rho$ is the density, and a harmonic time factor $\exp (-i \sigma t)$ is assumed implicitly in the dependent variables.

Assume the displacements are given in terms of potentials $\phi, \psi$ by

$$
\begin{aligned}
& \mu u=\mu^{\prime} \frac{\partial \phi}{\partial x^{\prime}}-\frac{\partial \psi}{\partial y^{\prime}}, \\
& \mu v=\mu^{\prime} \frac{\partial \phi}{\partial y^{\prime}}+\frac{\partial \psi}{\partial x^{\prime}},
\end{aligned}
$$

so that the usual isotropic linear stress-strain relations may be written in the form

$$
\begin{aligned}
& \tau_{x x}=\lambda^{\prime} \nabla_{1}^{2} \phi+2 \mu^{\prime} \frac{\partial^{2} \phi}{\partial x^{\prime 2}}-2 \frac{\partial^{2} \psi}{\partial x^{\prime} \partial y^{\prime}}, \\
& \tau_{x y}=2 \mu^{\prime} \frac{\partial^{2} \phi}{\partial x^{\prime} \partial y^{\prime}}+\frac{\partial^{2} \psi}{\partial x^{\prime 2}}-\frac{\partial^{2} \psi}{\partial y^{\prime 2}}, \\
& \tau_{y y}=\lambda^{\prime} \nabla_{1}^{2} \phi+2 \mu^{\prime} \frac{\partial^{2} \phi}{\partial y^{\prime 2}}-2 \frac{\partial^{2} \psi}{\partial x^{\prime} \partial y^{\prime}} .
\end{aligned}
$$

In these equations, $\nabla_{1}^{2}$ is the two-dimensional Laplacian operator, $\lambda^{\prime}, \mu^{\prime}$ are constants given in terms of the Lamé constants $\lambda, \mu$ by

$$
\lambda^{\prime}=\lambda /(\lambda+2 \mu), \quad \mu^{\prime}=\mu /(\lambda+2 \mu)
$$

so that $\lambda^{\prime}+2 \mu^{\prime}=1$.

Let $L$ be a typical constant length, and choose $x=x^{\prime} / L, y=y^{\prime} / L$, when from (2.1) and (2.3)

$$
\begin{aligned}
& \nabla^{2}\left(\frac{\partial \phi}{\partial x}-\frac{\partial \psi}{\partial y}\right)+k^{2}\left(\mu^{\prime} \frac{\partial \phi}{\partial x}-\frac{\partial \psi}{\partial y}\right)=0 \\
& \nabla^{2}\left(\frac{\partial \phi}{\partial y}+\frac{\partial \psi}{\partial x}\right)+k^{2}\left(\mu^{\prime} \frac{\partial \phi}{\partial y}+\frac{\partial \psi}{\partial x}\right)=0
\end{aligned}
$$


where the non-dimensional wave number $k$ is given by

$$
k^{2}=\rho \sigma^{2} L^{2} / \mu
$$

and $\nabla^{2}$ is the Laplacian in the $(x, y)$ coordinates. Clearly solutions of the pair of equations

$$
\begin{aligned}
\left(\nabla^{2}+\mu^{\prime} k^{2}\right) \phi & =0, \\
\left(\nabla^{2}+k^{2}\right) \psi & =0,
\end{aligned}
$$

are solutions of (2.5), and it is well known that for a given dynamic boundary value problem, solution of (2.6) and (2.7) is sufficient to obtain the complete solution of (2.5).

Now assume that $k \ll 1$, and let us postulate the perturbation expansions

$$
\begin{aligned}
& \phi=\phi_{0}+k \phi_{1}+k^{2} \phi_{2}+\ldots, \\
& \psi=\psi_{0}+k \psi_{1}+k^{2} \psi_{2}+\ldots
\end{aligned}
$$

In order to find the equations for $\phi_{i}, \psi_{i}$, however, it is necessary to substitute the perturbation expansions in the full equations (2.5). After comparing coefficients of powers of $k$, the generalized Cauchy-Riemann relations

$$
\begin{aligned}
& \nabla^{2}\left(\frac{\partial \phi_{i}}{\partial x}-\frac{\partial \psi_{i}}{\partial y}\right)=-\left(\mu^{\prime} \frac{\partial \phi_{i-2}}{\partial x}-\frac{\partial \psi_{i-2}}{\partial y}\right) \\
& \nabla^{2}\left(\frac{\partial \phi_{i}}{\partial y}+\frac{\partial \psi_{i}}{\partial x}\right)=-\left(\mu^{\prime} \frac{\partial \phi_{i-2}}{\partial y}+\frac{\partial \psi_{i-2}}{\partial x}\right)
\end{aligned}
$$

for $i=1,2,3 \ldots$, are obtained and the right-hand sides are zero when $i=1,2$. It follows that

$$
\begin{aligned}
& \nabla^{4} \phi_{i}=-\mu^{\prime} \nabla^{2} \phi_{i-2}, \quad i \geqslant 2, \\
& \nabla^{4} \psi_{i}=-\nabla^{2} \psi_{i-2}, \quad i \geqslant 2,
\end{aligned}
$$

and, for $i=1,2$, the functions $\phi_{i}, \psi_{i}$ satisfy the biharmonic equation

$$
\nabla^{4} \phi_{i}=0, \quad \nabla^{4} \psi_{i}=0, \quad i=1,2 .
$$

The case of plane elastostatics is obtained by taking the limit of (2.5), as $k \rightarrow 0$, leading to biharmonic equation for $\phi$ and $\psi$.

\section{Plane Elastostatics}

Standard derivations of the equations of plane elastostatics involve the Airy stress function (Muskhelishvili [3]; Sokolnikoff [6]). However, it is difficult to relate the Airy stress function to the displacement potentials of elastodynamics, and an alternative formulation of the equations of plane elastostatics is desirable in order to consider the diffraction problems posed in this paper. 
Let $z=x+i y, \bar{z}=x-i y$, and

$$
w=\phi+i \psi,
$$

where $\phi, \psi$ are given in (2.2), but with the frequency $\sigma=0$. Noting that

$$
2 \frac{\partial}{\partial z}=\frac{\partial}{\partial x}-i \frac{\partial}{\partial y}
$$

we may rewrite (2.5), with $k=0$, as the single equation

$$
\frac{\partial^{3} w}{\partial z \partial \bar{z}^{2}}=0
$$

which has as its general solution

$$
w=\bar{z} \Omega(z)+A(z)+\bar{B}(\bar{z}),
$$

where $\Omega(z), A(z), B(z)$ are arbitrary functions of $z$ which are analytic in the regions considered.

If $\chi(z)$ is any analytic function of $z$, and

$$
\chi(z)=\rho_{1}(x, y)+i \rho_{2}(x, y),
$$

where $\rho_{1}, \rho_{2}$ are conjugate, real harmonic functions, then direct substitution into (2.2) shows that if

$$
w^{*}=\phi^{*}+i \psi^{*}=\rho_{1}+i \mu^{\prime} \rho_{2}=\frac{1}{2}\left(1+\mu^{\prime}\right) \chi(z)+\frac{1}{2}\left(1-\mu^{\prime}\right) \bar{\chi}(\bar{z}),
$$

then $u=v \equiv 0$. In other words, if $w^{*}$ is expressed in terms of $\chi(z)$ by (3.4), then $w^{*}$ is an arbitrary "null solution" which has zero displacements and stresses everywhere. Now let

$$
\chi(z)=-2 B(z) /\left(1-\mu^{\prime}\right)
$$

and

$$
\int \omega(z) d z=A(z)+\frac{1}{2}\left(1+\mu^{\prime}\right) \chi(z) .
$$

Since we may subtract $w^{*}$ from (3.3) without changing the displacements and stress, a general expression for $w$ in terms of two arbitrary functions $\Omega(z), \omega(z)$ is

$$
w=\bar{z} \Omega(z)+\int \omega(z) d z .
$$

The following expressions may now be derived by substitution of (3.5) and (3.1) in (2.2) and (2.3)

$$
\begin{gathered}
\Theta=\tau_{x x}+\tau_{y y}=2\left(1-\mu^{\prime}\right) \nabla^{2} \phi=4\left(1-\mu^{\prime}\right)\left[\Omega^{\prime}(z)+\bar{\Omega}^{\prime}(\bar{z})\right], \\
\Phi=\tau_{x x}-\tau_{y y}+2 i \tau_{x y}=-8\left(1-\mu^{\prime}\right) \frac{\partial^{2} \phi}{\partial \bar{z}^{2}}=-4\left(1-\mu^{\prime}\right)\left[z \bar{\Omega}^{\prime \prime}(\bar{z})+\bar{\omega}^{\prime}(\bar{z})\right] .
\end{gathered}
$$


Also, let the complex displacement $D$ be given by

$$
\left(1-\mu^{\prime}\right) D=\mu(u+i v)
$$

and then

$$
D=\kappa \Omega(z)-z \bar{\Omega}^{\prime}(\bar{z})-\bar{\omega}(\bar{z})
$$

where $\kappa=\left(1+\mu^{\prime}\right) /\left(1-\mu^{\prime}\right)$.

The choice of $w$ in terms of the two functions in (3.5) is sufficient, but not unique. This particular choice, however, results in expressions (3.6) to (3.9) which are almost identical to the standard complex variable expressions derived by Muskhelishvili. The importance of the above derivation is that it is possible to determine directly the displacement potentials $\phi, \psi$ in terms of $\Omega, \omega$, while it is not obvious how this is to be done in the Muskhelishvili theory. Nevertheless, having derived (3.6) to (3.9), it is possible to use all existing results in the Muskhelishvili theory without substantial modifications. For instance, additional useful results may be derived as follows.

If $\Theta^{\prime}, \Psi^{\prime}$ are the complex stress combinations

$$
\Theta^{\prime}=\tau_{n n}+\tau_{s s}, \quad \Phi^{\prime}=\tau_{n n}-\tau_{s s}+2 i \tau_{n s},
$$

referred to rotated axes

$$
z^{\prime}=n+i s=z e^{-i \alpha},
$$

then it may be shown that

$$
\Theta^{\prime}=\Theta \text { and } \Phi^{\prime}=\Phi e^{-2 i \alpha} .
$$

An additional useful result derived in Appendix $\mathrm{A}$ is that the resultant force on an $\operatorname{arc} \Gamma$ is

$$
X+i Y=-2 i\left(1-\mu^{\prime}\right)\left[z \bar{\Omega}^{\prime}(\bar{z})+\Omega(z)+\bar{\omega}(\bar{z})\right]_{\Gamma},
$$

so that the condition that an $\operatorname{arc} \Gamma$ is free of stress is that

$$
z \bar{\Omega}^{\prime}(\bar{z})+\Omega(z)+\bar{\omega}(\bar{z})=\text { const. }
$$

on $\Gamma$.

Finally, it should also be noted that the choice

$$
\Omega_{D}(z)=0, \quad \omega_{D}(z)=C_{1}+i C_{2}
$$

gives a rigid body translation $D=-\left(C_{1}+i C_{2}\right)$, with the corresponding

$$
\phi_{D}=r\left(C_{1} \cos \theta-C_{2} \sin \theta\right), \quad \psi_{D}=r\left(C_{1} \sin \theta+C_{2} \cos \theta\right),
$$

while, for real $E$,

$$
\Omega_{R}=E i z, \quad \omega_{R}(z)=0
$$

correspond to the rigid body rotation

$$
D=(\kappa-1) E i z .
$$




\section{Diffraction by a circular cylindrical hole}

Let there be a circular hole for $x^{\prime 2}+y^{\prime 2}<a^{2}$, and the circle $x^{\prime 2}+y^{\prime 2}=a^{2}$ is assumed free of stress. An incident plane $P$ wave is assumed to be of form

$$
\phi_{I}=\exp \left[i\left(K x^{\prime}-\sigma t\right)\right], \quad \psi_{I}=0,
$$

where $K^{2}=\rho \sigma^{2} /(\lambda+2 \mu)$.

The scattered waves due to diffraction by the cylinder have the general form, taking symmetry into account,

$$
\phi_{s}=\sum_{j=1}^{\infty} A_{j} H_{j}^{(1)}\left(K r^{\prime}\right) \cos \theta, \quad \psi_{s}=\sum_{j=0}^{\infty} B_{j} H_{j}^{(1)}\left(K r^{\prime} / \sqrt{ } \mu^{\prime}\right) \sin j \theta,
$$

where $\left(r^{\prime}, \theta\right)$ are polar coordinates with $r^{\prime}=\left(x^{\prime 2}+y^{\prime 2}\right)^{\frac{1}{2}}, H_{j}^{(1)}$ is the Hankel function of the first kind, and the $A_{j}, B_{j}$ are to be determined so that $\tau_{r r}=\tau_{r \theta}=0$ on the cylinder surface.

Make the coordinate transformation

$$
z=x+i y, \quad x=x^{\prime} / a, \quad y=y^{\prime} / a, \quad a K=k_{1},
$$

where $k_{1}^{2}=\mu^{\prime} k^{2}$, as in Section 2. Assuming $k_{1} \ll 1$, it may be shown from (4.1) and (2.3) that the stresses due to the incident wave near $|z|=1$ are given by

$K^{-2} \tau_{x x}=-\left(1+i k_{1} x\right)+O\left(k^{2}\right), \quad \tau_{x y}=0, \quad K^{-2} \tau_{y y}=-\lambda^{\prime}\left(1+i k_{1} x\right)+O\left(k^{2}\right)$,

so that in polar coordinates, on $|z|=1$, the stress due to the incident wave is given by

where

$$
K^{-2}\left(\tau_{r r}+i \tau_{r \theta}\right)=\tau_{I}=\tau_{I}^{(0)}+i k_{1} \tau_{I}^{(1)}+O\left(k^{2}\right)
$$

and

$$
\tau_{I}^{(0)}=-\left(1-\mu^{\prime}+\mu^{\prime} e^{-2 i \theta}\right)
$$

$$
\tau_{I}^{(1)}=-\frac{1}{2}\left[\left(1-\mu^{\prime}\right) e^{i \theta}+e^{-i \theta}+\mu^{\prime} e^{-3 i \theta}\right]
$$

Noting that the change of scale gives a factor $a^{2}$ in the formulae for the complex stresses, direct substitution in $(3.6,3.7,3.10,3.12)$ shows that

$$
\Omega_{0}(z)=-\alpha_{0} z^{-1}, \quad \omega_{0}(z)=\alpha_{1} z^{-1}-\alpha_{0} z^{-3}
$$

where

$$
\alpha_{0}=\frac{1}{2} \mu^{\prime} k_{1}^{2} /\left(1-\mu^{\prime}\right), \quad \alpha_{1}=\frac{1}{2} k_{1}^{2},
$$

gives the solution of the static problem in $|z|>1$ in which

$$
\tau_{r r}+i \tau_{1 \theta}=-K^{2} \tau_{I}^{(0)}
$$

on $|z|=1$. Similarly, the functions

$$
\Omega_{1}(z)=\beta_{0} \log z-\beta_{1} z^{-2}, \quad \omega_{1}(z)=-\kappa \beta_{0} \log z-\beta_{2} z^{-4},
$$

where

$$
\beta_{0}=\frac{1}{8} k_{1}^{2}, \quad 2 \beta_{1}=\beta_{2}=\frac{1}{4} \mu^{\prime} k_{1}^{2} /\left(1-\mu^{\prime}\right)
$$


yield the solution of the static problem in $|z|>1$ in which

$$
\tau_{r r}+i \tau_{r \theta}=-K^{2} \tau_{I}^{(1)}
$$

on $|z|=1$. Note that the coefficient $\kappa \beta_{0}$ of $\log z$ in $\omega_{1}(z)$ in (4.11) is chosen to ensure that the complex displacement $D$ in (3.9) is single valued for complete circuits around $z=0$.

Noting that

$$
\phi+i \psi=\bar{z} \Omega(z)+\int \omega(z) d z,
$$

the field in the "inner" region near the obstacle is now determined in the form

$$
\begin{aligned}
& \phi=\phi_{0}+i k_{1} \phi_{1}+\phi^{*}+O\left(k^{2}\right), \\
& \psi=\psi_{0}+i k_{1} \psi_{1}+\psi^{*}+O\left(k^{2}\right),
\end{aligned}
$$

where $\phi_{0}, \psi_{0}$ are found from (4.8) to be, in polar coordinates,

$$
\phi_{0}=\alpha_{1} \log r-\alpha_{0}\left(1-2 r^{-2}\right) \cos 2 \theta, \quad \psi_{0}=\alpha_{1} \theta+\alpha_{0}\left(1-2 r^{-2}\right),
$$

and $\phi_{1}, \psi_{1}$ are found from (4.11) to be

$$
\begin{aligned}
& \phi_{1}=\left[\beta_{0}(1-\kappa) r \log r+\kappa \beta_{0} r\right] \cos \theta+(1+\kappa) \beta_{0} r \theta \sin \theta-r^{-1}\left(\beta_{1}-\beta_{2} / 3 r^{2}\right) \cos 3 \theta \\
& \psi_{1}=\left[-\beta_{0}(1+\kappa) r \log r+\kappa \beta_{0} r\right] \sin \theta+(1-\kappa) \beta_{0} r \theta \cos \theta+r^{-1}\left(\beta_{1}-\beta_{2} / 3 r^{2}\right) \sin 3 \theta
\end{aligned}
$$

and $\phi^{*}, \psi^{*}$ are arbitrary at this stage, but $\phi^{*}$ and $\psi^{*} / \mu^{\prime}$ are conjugate harmonic functions, as in (3.4).

The method of asymptotic matching consists of finding a general solution of the exact equations in an "outer region", and matching the result, as $r \rightarrow 0$, with the limit, as $r \rightarrow \infty$, of the solution of the approximate equations with the correct boundary condition in an "inner region". Here the general solution in the outer region is given in (4.2). We make the transformation (4.3) to the scattered field in (4.2), and match the limit, as $r \rightarrow 0$, with the limit, as $r \rightarrow \infty$, of the solution in the inner region in (4.15). Obviously, comparing coefficients of $\cos n \theta, \sin n \theta$, the required outer solution is of the form

$$
\phi_{s}=\phi_{0 s}+\phi_{1 s}, \quad \psi_{s}=\psi_{0 s}+\psi_{1 s},
$$

where

$$
\begin{aligned}
& \phi_{0 s}=A_{0} H_{0}^{(1)}\left(k_{1} r\right)+A_{2} H_{2}^{(1)}\left(k_{1} r\right) \cos 2 \theta, \\
& \psi_{0 s}=B_{2} H_{2}^{(1)}(k r) \sin 2 \theta \\
& \phi_{1 s}=A_{1} H_{1}^{(1)}\left(k_{1} r\right) \sin \theta+A_{3} H_{3}^{(1)}\left(k_{1} r\right) \sin 3 \theta \\
& \psi_{1 s}=B_{1} H_{1}^{(1)}(k r) \sin \theta+B_{3} H_{3}^{(1)}(k r) \sin 3 \theta
\end{aligned}
$$


Consequently, as $r \rightarrow 0$, we obtain, using the approximations to the Hankel functions listed in Appendix C,

$$
\begin{aligned}
& \phi_{0 s}=\frac{2 i}{\pi} A_{0} \log r-\frac{i}{\pi} A_{2}\left(1+\frac{4}{k_{1}^{2} r^{2}}\right) \cos 2 \theta+O\left(r^{2} \log r\right), \\
& \psi_{0 s}=-\frac{i}{\pi} B_{2}\left(1+\frac{4}{k^{2} r^{2}}\right) \sin 2 \theta+O\left(r^{2} \log r\right) .
\end{aligned}
$$

These expressions are compared with those in (4.16) and we find that

$$
\phi_{0 s}=\phi_{0}+\phi_{0}^{*}, \quad \psi_{0 s}=\psi_{0}+\psi_{0}^{*},
$$

where $\phi_{0}^{*}, \psi_{0}^{*} / \mu^{\prime}$ are conjugate harmonic functions if

$$
A_{0}=\frac{1}{4} \pi i k_{1}^{2}\left(1-\mu^{\prime}\right) / \mu^{\prime}, \quad A_{2}=-B_{2}=-\frac{1}{2} \pi i k_{1}^{2} \mu^{\prime} /\left(1-\mu^{\prime}\right),
$$

and

$$
\begin{aligned}
& \phi_{0}^{*}=X_{0} \log r+X_{2} \cos 2 \theta / r^{2}, \\
& \psi_{0}^{*}=\mu^{\prime} X_{0} \theta-\mu^{\prime} X_{2} \sin 2 \theta / r^{2},
\end{aligned}
$$

where $X_{0}=-\alpha_{1} / \mu^{\prime}, X_{2}=4 \alpha_{0} / k_{1}^{2}$, and $\alpha_{0}, \alpha_{1}$ are given in (4.9).

Similarly, as $r \rightarrow 0$

$$
\begin{aligned}
i \pi \phi_{1 s}= & {\left[\frac{2}{k_{1} r}+\frac{1}{2} k_{1} r\left(b-2 \log k_{1}-2 \log r\right)\right] A_{1} \cos \theta } \\
& +\left[\frac{16}{\left(k_{1} r\right)^{3}}+\frac{2}{\left(k_{1} r\right)}+\frac{k_{1} r}{4}\right] A^{3} \cos 3 \theta+o\left(r^{3} \log r\right) \\
i \pi \psi_{1 s}= & {\left[\frac{2}{k r}+\frac{1}{2} k r(b-2 \log k-2 \log r)\right] B_{1} \sin \theta } \\
& +\left[\frac{16}{(k r)^{3}}+\frac{2}{k r}+\frac{k r}{4}\right] B_{3} \sin 3 \theta+o\left(r^{3} \log r\right)
\end{aligned}
$$

where $b=1-2 \gamma+i \pi+2 \log 2$, and $\gamma$ is Euler's constant. Then, matching as before,

$$
\phi_{1 s}=\phi_{1}+\phi_{1}^{*}+\phi_{D}, \quad \psi_{1 s}=\psi_{1}+\psi_{1}^{*}+\psi_{D},
$$

where $\phi_{1}, \psi_{1}$ are given in (4.17), and it is necessary to introduce the "null potentials"

$$
\begin{aligned}
& \phi_{1}^{*}=X_{1}(r \cos \theta \log r-r \theta \sin \theta)+\left(Y_{1} r^{-1}+Z_{1} r\right) \cos \theta+X_{3} r^{-3} \cos 3 \theta, \\
& \psi_{1}^{*} / \mu=X_{1}(r \sin \theta \log r+r \theta \cos \theta)-\left(Y_{1} r^{-1}-Z_{1} r\right) \cos \theta-X_{3} r^{-3} \sin 3 \theta,
\end{aligned}
$$

and the rigid body translation, as in (3.15),

$$
\phi_{D}=C_{1} r \cos \theta, \quad \psi_{D}=C_{1} r \sin \theta,
$$

in order to complete the matching. It follows that

$$
A_{1} k_{1}=-B_{1} k=2 \pi k_{1} \beta_{0}
$$




$$
A_{3}=-B_{3} k_{1} / k=\frac{1}{2} \pi k_{1}^{2} \beta_{1},
$$

where $\beta_{0}, \beta_{1}$ are given in (4.12), provided that

$$
\begin{aligned}
\left(1-\mu^{\prime}\right) X_{1} & =2 \beta_{0}, \quad k_{1} Y_{1}=-4 i \beta_{0}, \quad k_{1} X_{3}=-8 i \beta_{1}, \\
2 \pi i\left(1-\mu^{\prime}\right) Z_{1} & =\left(k_{1}-k\right) b+2 k \log k-2 k_{1} \log k_{1}, \\
2 \pi i\left(1-\mu^{\prime}\right)\left(C_{1}+\kappa \beta_{0}\right) & =2 k \log k-2 \mu^{\prime} k_{1} \log k_{1}+\left(k-\mu^{\prime} k_{1}\right) b .
\end{aligned}
$$

\section{Discussion}

Given an incident wave of the form in (4.1), the diffracted wave is

$$
\begin{aligned}
& \phi_{s}=\sum_{n=0}^{2} A_{n} H_{n}^{(1)}\left(K r^{\prime}\right) \cos n \theta, \\
& \psi_{s}=\sum_{n=1}^{2} B_{n} H_{n}^{(1)}\left(K r^{\prime} / \mu^{\prime}\right) \sin n \theta,
\end{aligned}
$$

where the coefficients $A_{n}, B_{n}$ are determined in (4.22), (4.26), and terms of $O\left(k_{1}^{4}\right)$ are neglected. These results agree with those derived in Appendix B by approximating expressions computed from the exact solution. Some numerical results for moderately large $k$ are given in Table 1 , in the case $\mu^{\prime}=0.33$.

Although some care is needed to take into account the null potentials $\phi^{*}, \psi^{*}$ and rigid body displacements, the techniques described in this are suitable for obtaining asymptotic approximations for low frequency scattering of elastic waves in two dimensions by cylindrical obstacles. The method is suitable for any obstacle with a cross-section that can be mapped conformally onto a circle. Investigations of some specific examples of scattering by non-circular cylindrical cavities are at an advanced stage.

\section{TABLE 1}

Modulus and argument of $A_{n}$, obtained from Appendix B, compared with the asymptotically determined $A_{n}^{*}$, when $\mu^{\prime}=0.33, n=1,2$ and $k_{1}=0.1,0.2$. The error is of the order $k^{2}$ in each case

\begin{tabular}{ccccccc}
\hline$n$ & $k_{1}$ & $k$ & $\left|A_{n}\right|$ & $\left|A_{n}^{*}\right|$ & $\arg A_{n}$ & $\arg A_{n}^{*}$ \\
\hline 1 & 0.1 & 0.174 & 0.0077 & 0.0079 & 0.015 & 0 \\
1 & 0.2 & 0.348 & 0.0029 & 0.0031 & 0.058 & 0 \\
2 & 0.1 & 0.174 & 0.0081 & 0.0079 & -1.53 & -1.57 \\
2 & 0.2 & 0.348 & 0.0033 & 0.0031 & -1.4 & -1.57 \\
\hline
\end{tabular}




\section{Appendix A. The resultant force on an arc}

Let $\mathbf{n}$ be the normal and $\mathbf{s}$ the tangent to the arc $\Gamma$ in the sense of (3.11), as in Figure 1. If $X, Y$ are the resultant forces on the arc in the $x, y$ directions, it may be shown that

$$
X+i Y=\int_{\Gamma}\left(\tau_{n n}+i \tau_{n s}\right) e^{i x} d s
$$

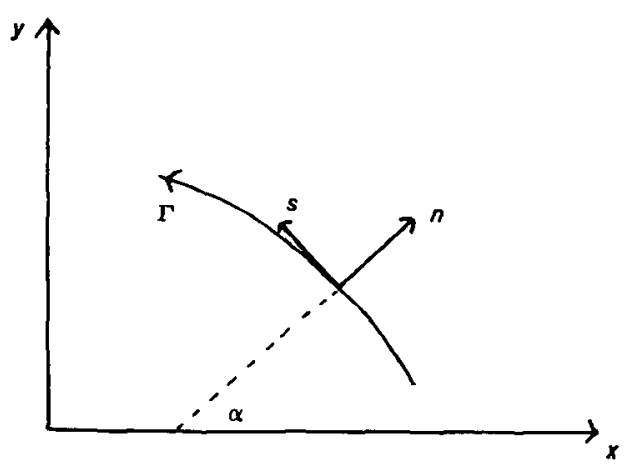

Fig. 1. Illustrating the co-ordinates $\mathbf{n}$ and $\mathbf{s}$.

where $\alpha$ is the angle $\mathbf{n}$ makes with the $x$ axis. Using (3.10) and (3.12),

$$
X+i Y=\frac{1}{2} \int_{\Gamma}\left(\Theta e^{i \alpha}+\Phi e^{-i \alpha}\right) d s
$$

which, together with (3.6), (3.7) and $\left(3.11^{\alpha}\right.$, yields the result

$$
\begin{aligned}
X+i Y & =-4 i\left(1-\mu^{\prime}\right) \int_{\Gamma}\left(\frac{\partial^{2} \Phi}{\partial z \partial \bar{z}} d z+\frac{\partial^{2} \Phi}{\partial \bar{z}^{2}} d \bar{z}\right) \\
& =-4 i\left(1-\mu^{\prime}\right) \int_{\Gamma} d\left(\frac{\partial \Phi}{\partial \bar{z}}\right)
\end{aligned}
$$

From (3.5) and (3.1),

$$
\frac{\partial \Phi}{\partial \bar{z}}=\frac{1}{2}\left[z \bar{\Omega}^{\prime}(\bar{z})+\Omega(z)+\bar{\omega}(\bar{z})\right]
$$

The result (3.13) follows immediately from (A3) and (A4). 


\section{Appendix B. Scattering by a hollow circular cylinder}

The incident wave in (4.1) may be written as an expansion in Bessel functions

$$
\phi_{I}=J_{0}\left(k_{1} r\right)+2 \sum_{n=1}^{\infty}(i)^{n} J_{n}\left(k_{1} r\right) \cos n \theta
$$

in terms of the non-dimensional quantities $r=r^{\prime} / a, k_{1}=k /\left(\mu^{\prime}\right)^{\frac{1}{2}}=a K$. The scattered wave is

$$
\phi_{s}=\sum_{n=0}^{\infty} A_{n} H_{n}\left(k_{1} r\right) \cos n \theta, \quad \psi_{s}=\sum_{n=1}^{\infty} B_{n} H_{n}(k r) \sin n \theta,
$$

where $H_{n}$ is the Hankel function of the first kind (usually written as $H_{n}^{(1)}=J_{n}+i Y_{n}$ ) when the implicit time factor is $\exp (-i \sigma t)$. The boundary conditions $\tau_{r r}=\tau_{r \theta}=0$ at $r=1$ may be written as

$$
\begin{gathered}
{\left[\nabla^{2}-\frac{2 \mu^{\prime}}{r}\left(\frac{\partial}{\partial r}+\frac{1}{r} \frac{\partial^{2}}{\partial \theta^{2}}\right)\right]\left(\phi_{I}+\phi_{s}\right)-\frac{2}{r}\left(\frac{\partial}{\partial r}-\frac{1}{r}\right) \frac{\partial \psi_{s}}{\partial \theta}=0,} \\
\frac{2 \mu^{\prime}}{r} \frac{\partial}{\partial \theta}\left(\frac{\partial}{\partial r}-\frac{1}{r}\right)\left(\phi_{I}+\phi_{s}\right)+\left[\nabla^{2}-\frac{2}{r}\left(\frac{\partial}{\partial r}+\frac{1}{r} \frac{\partial^{2}}{\partial \theta^{2}}\right)\right] \psi_{s}=0,
\end{gathered}
$$

at $r=1$. Substitution of (B1, B2) into (B3, B4) yields, on comparison of coefficients of $\cos n \theta, \sin n \theta$, and the use of the Bessel function recurrence relations,

$$
\begin{aligned}
& T_{11}(n) A_{n}+T_{12}(n) B_{n}=S_{1}(n) \\
& T_{21}(n) A_{n}+T_{22}(n) B_{n}=S_{2}(n)
\end{aligned}
$$

where

$$
\begin{aligned}
T_{11} & =\left(k_{1}^{2}-2 \mu^{\prime} n-2 \mu^{\prime} n^{2}\right) H_{n}\left(k_{1}\right)+2 \mu^{\prime} k_{1} H_{n-1}\left(k_{1}\right), \\
T_{12} & =-2 n\left[(n+1) H_{n}(k)-k H_{n-1}(k)\right], \\
T_{21} & =-2 \mu^{\prime} n\left[(n+1) H_{n}\left(k_{1}\right)-k_{1} H_{n-1}\left(k_{1}\right)\right], \\
T_{22} & =\left(k^{2}-2 n-2 n^{2}\right) H_{n}(k)+2 k H_{n-1}(k), \\
S_{1} & =-\varepsilon_{n}\left[\left(k_{1}^{2}+2 \mu^{\prime} n-2 \mu^{\prime} n^{2}\right) J_{n}\left(k_{1}\right)-2 \mu^{\prime} k_{1} J_{n+1}\left(k_{1}\right)\right], \\
S_{2} & =-2 n \mu^{\prime} \varepsilon_{n}\left[(n-1) J_{n}\left(k_{1}\right)-k_{1} J_{n+1}\left(k_{1}\right)\right],
\end{aligned}
$$

and $\varepsilon_{n}=2(i)^{n}$ for $n \geqslant 1$, with $\varepsilon_{0}=1$. Equations (B5) may be solved to find $A_{n}, B_{n}$, but we are interested here in their approximate solution for small $k$. 
We make use of the approximations for the Bessel functions given in Appendix C. Noting that $H_{-1}=-H_{1}$, we find from (B5a) that for $n=0$,

$$
A_{0}=\frac{1}{4} \pi i k_{1}^{2}\left(1-\mu^{\prime}\right) / \mu^{\prime}+O\left(k_{1}^{2} \log k_{1}\right),
$$

while (B5b) is then satisfied identically. For $n \geqslant 1$, it can be shown the lowest order terms in (B5a) and (B5b) are consistent only if, for small $k$,

$$
\mu^{\prime} k^{n} A_{n}+k_{1}^{n} B_{n}=O\left(k^{n+2}\right), \quad n \geqslant 1 .
$$

Subtraction of (B5b) from (B5a) yields the result that for $n \geqslant 2$

$$
A_{n}=\frac{i \pi \varepsilon_{n} \mu^{\prime}(n-1)}{\left(1-\mu^{\prime}\right)\{(n-1) !\}^{2}}\left(\frac{k_{1}}{2}\right)^{2 n-2}+O\left(k_{1}^{2 n}\right) .
$$

In particular,

$$
A_{2}=-B_{2}=-\frac{1}{2} \pi j \mu^{\prime} k_{1}^{2} /\left(1-\mu^{\prime}\right)+O\left(k_{1}^{4}\right) .
$$

When $n=1$, some terms vanish, and the result is

$$
A_{1}=-k B_{1} / k_{1}=\frac{1}{4} \pi k_{1}^{2}+O\left(k_{1}^{4} \log k_{1}\right) \text {. }
$$

\section{Appendix C. Asymptotic expansions for the Bessel functions}

For small arguments, the required asymptotic forms for the Bessel functions are (Abramowitz and Stegan, [1]),

$$
J_{n}(\xi)=\xi^{n} / 2^{n} n !+O\left(\xi^{n+2}\right), \quad n \geqslant 0,
$$

and, for $n \geqslant 2$,

$$
H_{n}(\xi)=-\frac{i(n-2) !}{\pi}\left(\frac{2}{\xi}\right)^{n}\left[n-1+\frac{\xi^{2}}{8}\right]+O\left(\xi^{4-n}\right) .
$$

For $n=0,1$, we have

$$
\begin{aligned}
& H_{1}(\xi)=-\frac{2 i}{\pi \xi}+\left[2 \gamma-\pi i-1+2 \log \left(\frac{1}{2} \xi\right)\right] \frac{i \xi}{2 \pi}+O\left(\xi^{3} \log \xi\right), \\
& H_{0}(\xi)=1+\frac{2}{\pi} i\left[\gamma+\log \left(\frac{1}{2} \xi\right)\right]+O\left(\xi^{2} \log \xi\right)
\end{aligned}
$$

where $\gamma$ is Euler's constant.

\section{References}

[1] M. Abramowitz and I. A. Stegun, Handbook of mathematical functions (New York: Dover, 1965).

[2] P. L. Lutz, Some effects of topography on Rayleigh waves (University of Western Australia:

Ph.D. thesis, 1977). 
[3] N. I. Muskhelishvili, Some basic problems of the mathematical theory of elasticity, translated by J. R. M. Radok (Nordhoff, 1953).

[4] J. W. S. Rayleigh, The theory of sound (New York: Dover, revised second edition, 1945), Vol. 1, p. 343.

[5] F. J. Sabina and J. R. Willis, Geophys. J. Roy. Astr. Soc. 42 (1975), 685.

[6] I. S. Sokolnikoff, Mathematical theory of elasticity (New York: McGraw-Hill, second edition, 1956), Chapter 5.

[7] E. Steinberg, Arch. Rat. Mech. Anal. 6 (1960), 34.

School of Mathematics

University of N.S.W.

Kensington, N.S.W. 2033

Australia 